\title{
Resting state functional connectivity alterations in primary lateral sclerosis
}

\author{
Federica Agosta ${ }^{a}$, Elisa Canu ${ }^{\mathrm{a}}$, Alberto Inuggi ${ }^{\mathrm{a}}$, Adriano Chiò ${ }^{\mathrm{d}}$, Nilo Riva ${ }^{\mathrm{b}}$, \\ Vincenzo Silani ${ }^{\mathrm{e}, \mathrm{f}}$, Andrea Calvo ${ }^{\mathrm{d}}$, Stefano Messina ${ }^{\mathrm{e}, \mathrm{f}}$, Andrea Falini ${ }^{\mathrm{c}}$, Giancarlo Comi ${ }^{\mathrm{b}}$, \\ Massimo Filippi ${ }^{\mathrm{a}, \mathrm{b}, *}$ \\ ${ }^{a}$ Neuroimaging Research Unit, Institute of Experimental Neurology, Division of Neuroscience, San Raffaele Scientific Institute, Vita-Salute San Raffaele \\ University, Milan, Italy \\ ${ }^{\mathrm{b}}$ Department of Neurology, San Raffaele Scientific Institute, Vita-Salute San Raffaele University, Milan, Italy \\ ${ }^{\mathrm{c}}$ Department of Neuroradiology, CERMAC, San Raffaele Scientific Institute, Vita-Salute San Raffaele University, Milan, Italy \\ d 'Rita Levi Montalcini' Department of Neuroscience, University of Torino, Torino, Italy \\ e Department of Neurology and Laboratory of Neuroscience, IRCCS Istituto Auxologico Italiano, Milan, Italy \\ ${ }^{\mathrm{f}}$ Department of Pathophysiology and Transplantation, "Dino Ferrari" Center, Università degli Studi di Milano, Milan, Italy
}

\section{A R T I C L E I N F O}

\section{Article history:}

Received 21 May 2013

Received in revised form 11 September 2013

Accepted 19 September 2013

\section{Keywords:}

Primary lateral sclerosis

Resting state functional MRI

Functional connectivity

Sensorimotor network

Diffusion tensor MRI tractography

\begin{abstract}
A B S T R A C T
Resting state functional connectivity of the sensorimotor and extramotor brain networks was studied in 24 patients with primary lateral sclerosis (PLS) relative to 26 healthy controls. The relationships of RS functional connectivity with patient clinical and cognitive status and white matter tract damage (i.e., corticospinal tracts, corpus callosum, and superior longitudinal fasciculus) were investigated. Compared with controls, PLS patients showed an increased functional connectivity within the sensorimotor, frontal, and left frontoparietal networks spanning the pre- and postcentral, medial and dorsal frontal, insular, and superior temporal regions. Patients with more severe physical disability and a more rapid rate of disease progression had increased sensorimotor connectivity values. The increased functional connectivity within the frontal network was associated with executive dysfunction. In addition, higher functional connectivity correlated with greater structural damage to network-specific white matter tracts. This study shows clinically meaningful increased resting state functional connectivity in PLS.
\end{abstract}

(c) 2013 Elsevier Inc. All rights reserved.

\section{Introduction}

Primary lateral sclerosis (PLS) is a rare disorder characterized by an isolated degeneration of the upper motor neurons (UMN) with a lack of clinical signs of lower motor neuron (LMN) impairment (Pringle et al., 1992). PLS presents usually in the sixth decade of life with an insidious onset of a symmetric, slowly progressive spastic paresis, usually beginning in the lower extremities and eventually evolving into a tetrapyramidal syndrome with marked pseudobulbar features (Pringle et al., 1992). Clinical studies have estimated that $2 \%$ to $5 \%$ of patients seen in adult neuromuscular clinics are diagnosed with PLS (Le Forestier et al., 2001; Pringle et al., 1992). Early in the disease course, PLS is

\footnotetext{
* Corresponding author at: Neuroimaging Research Unit, Institute of Experimental Neurology, Division of Neuroscience, San Raffaele Scientific Institute, VitaSalute, San Raffaele University, Via Olgettina, 60, 20132 Milan, Italy. Tel.: +39 (0)2 26433033; fax: +39 (0)2 26435972.

E-mail address: m.filippi@hsr.it (M. Filippi).
}

difficult to distinguish from the predominant UMN amyotrophic lateral sclerosis (ALS) phenotype (Chio et al., 2011). However, PLS has a slower rate of progression and a more benign prognosis than ALS, with a survival of more than 10 years from symptom onset (Gordon et al., 2006; Kuipers-Upmeijer et al., 2001; Le Forestier et al., 2001).

Like ALS, PLS is a clinical diagnosis without established supportive biomarkers. On structural magnetic resonance imaging (MRI), atrophy of the primary motor cortices (PMC) was found in PLS patients (Kuipers-Upmeijer et al., 2001; Tartaglia et al., 2009). Diffusion tensor (DT) MRI shows a more severe white matter (WM) involvement of the corticospinal tracts (CST) and motor transcallosal fibers in PLS compared to those in ALS patients (Agosta et al., 2013b; Ciccarelli et al., 2009; Iwata et al., 2011; Muller et al., 2012; Unrath et al., 2010; van der Graaff et al., 2011).

Analysis of resting state (RS) functional MRI (fMRI) reveals the temporal correlation between the low-frequency spontaneous fluctuations in the resting brain. This allows identification of functionally distinct networks that are related to specific sensory, 
motor, and cognitive brain functions (Smith et al., 2009). RS fMRI alterations have been observed in the motor network of ALS patients (Agosta et al., 2011; Douaud et al., 2011; Jelsone-Swain et al., 2011; Mohammadi et al., 2009; Tedeschi et al., 2010; Verstraete et al., 2010). Previous studies in ALS have also described functional connectivity abnormalities extending well beyond the PMC (Agosta et al., 2013a; Douaud et al., 2011; Luo et al., 2012; Mohammadi et al., 2009; Tedeschi et al., 2010).

In the present study, we investigated RS functional connectivity of the sensorimotor and extramotor brain networks (i.e., default mode network [DMN], frontal network, left and right frontoparietal networks) in patients with PLS, and explored whether the RS fMRI alterations are related to patient clinical and cognitive status and WM structural connectivity breakdown.

\section{Methods}

\subsection{Subjects}

Patients with sporadic PLS (Pringle et al., 1992) were recruited at 3 tertiary referral Motor Neuron Disease (MND) Clinics in Northern Italy (San Raffaele Scientific Institute and "Vita-Salute" University, Milan; University of Turin, Turin; Università degli Studi di Milano, Milan). All patients had no evidence of acute or chronic denervation on repeated electromyographical (EMG) examinations and had had their symptoms for at least 3 years. To be eligible, subjects had to meet the following criteria: no family history of MND, no clinical diagnosis of frontotemporal dementia (Rascovsky et al., 2011), age at onset $\geq 40$ years, no mutations of major genes related to hereditary spastic paraparesis (i.e., SPG3A, SPG4, SPG7, SPG11) or any other major systemic, psychiatric, or neurological illnesses, no history of substance abuse, and no other causes of focal or diffuse brain damage, including strokes, lacunae, and other evidence of cerebrovascular disease at routine MRI scans. Within 48 hours from MRI, functional status was assessed using the ALS Functional Rating Scale (ALSFRS-r) (Cedarbaum et al., 1999). A quantitative assessment of clinical UMN involvement was based on the UMN score (Turner et al., 2004). The rate of disease progression at study entry was calculated as: (48 - ALSFRS-r score)/ time from symptom onset (Ellis et al., 1999). Healthy controls were recruited among spouses of patients and by word of mouth, underwent a neurological evaluation, and were included in the study only if the results were normal. A total of 24 PLS patients and 26 healthy controls were enrolled consecutively (Table 1 ). The study was approved by the local ethical committees, and written informed consent from all subjects was obtained before study initiation.

\subsection{Neuropsychological assessment}

Neuropsychological assessment was performed by an experienced neuropsychologist blinded to the MRI results, and evaluated the following: global cognition with the Mini Mental State Examination (Folstein et al., 1975); executive function with the semantic and phonemic fluency (Novelli et al., 1986) and the fluency indices (controlling for individual variations in motor disabilities) (Abrahams et al., 2000), Stroop test, Wisconsin Card Sorting Test (WCST) (Laiacona et al., 2000), digit span backward (Orsini et al., 1987), and Cognitive Estimation Test (Della Sala et al., 2003); reasoning and abstraction abilities with the Raven coloured progressive matrices and Weigl test (Basso et al., 1987; Spinnler and Tognoni, 1987); verbal memory with the digit span forward (Orsini et al., 1987) and Rey's word list (Carlesimo et al., 1996); and language with the BADA oral naming of objects and actions (Miceli et al., 1994). Scores on neuropsychological tests were age, sex, and education corrected by using normative values.
Table 1

Demographic, clinical, and structural MRI features of PLS patients and controls

\begin{tabular}{lccc}
\hline & Healthy controls & PLS & $p$ \\
\hline $\mathrm{N}$ & 26 & 24 & \\
Age at MRI (y) & $63.5 \pm 10.0(43-79)$ & $62.8 \pm 8.8(43-79)$ & 0.92 \\
Sex (female) & $13(50 \%)$ & $12(50 \%)$ & 1.00 \\
Disease duration & & $102.0 \pm 58.4(38-247)$ & \\
$\quad$ (mo) & & \\
ALSFRS-r & & $13.7 \pm 5.7(22-42)$ & \\
UMN score & & $0.15 \pm 0.11(0.03-0.4)$ & \\
Disease & & & \\
$\quad$ progression rate & & $0.5 \pm 0.8(0-4)$ & 0.21 \\
WM lesion load & $0.9 \pm 1.2(0-5)$ & & \\
$\quad($ mL) & & & 0.02 \\
R CST FA & $0.53 \pm 0.04(0.4-0.6)$ & $0.50 \pm 0.04(0.4-0.6)$ & 0.004 \\
L CST FA & $0.53 \pm 0.03(0.5-0.6)$ & $0.51 \pm 0.03(0.4-0.6)$ & 0.74 \\
R SLF FA & $0.43 \pm 0.03(0.4-0.5)$ & $0.43 \pm 0.02(0.4-0.5)$ & 0.94 \\
L SLF FA & $0.44 \pm 0.03(0.4-0.5)$ & $0.43 \pm 0.02(0.4-0.5)$ & 0.94 \\
CC FA & $0.52 \pm 0.03(0.5-0.6)$ & $0.50 \pm 0.03(0.4-0.6)$ & 0.55 \\
CC-genu FA & $0.46 \pm 0.04(0.4-0.5)$ & $0.47 \pm 0.02(0.4-0.5)$ & 0.50 \\
CC-body FA & $0.48 \pm 0.04(0.4-0.6)$ & $0.46 \pm 0.03(0.4-0.5)$ & 0.06 \\
CC-splenium FA & $0.54 \pm 0.03(0.5-0.6)$ & $0.54 \pm 0.03(0.5-0.6)$ & 0.61 \\
CC-PMC FA & $0.47 \pm 0.03(0.4-0.5)$ & $0.41 \pm 0.03(0.3-0.5)$ & $<0.001$ \\
CC-premotor FA & $0.43 \pm 0.03(0.4-0.5)$ & $0.43 \pm 0.03(0.4-0.5)$ & 0.65 \\
CC-SMA FA & $0.49 \pm 0.03(0.4-0.5)$ & $0.44 \pm 0.04(0.4-0.5)$ & $<0.001$ \\
\hline
\end{tabular}

Values are mean \pm standard deviation (range) or number (\%). $p$ Values refer to Mann-Whitney $U$ test or Fisher exact test, as appropriate. Disease progression rate: (48-ALSFRS-r score)/time from symptom onset.

Key: ALSFRS-r, ALS Functional Rating Scale-Revised; CC, corpus callosum; CST, corticospinal tract; FA, fractional anisotropy; L, left; MRI, magnetic resonance imaging; PLS, primary lateral sclerosis; PMC, primary motor cortex; R, right; SLF, superior longitudinal fasciculus; SMA, supplementary motor area; UMN, upper motor neuron.

\subsection{MRI acquisition}

Brain MRI scans were obtained using a 3.0 T scanner (Intera; Philips Medical Systems, Best, the Netherlands). A T2*-weighted single-shot echo planar imaging (EPI) sequence for RS fMRI was acquired from all subjects (repetition time $[\mathrm{TR}]=3000 \mathrm{~ms}$, echo time $[\mathrm{TE}]=35 \mathrm{~ms}$, flip angle $=90^{\circ}$, field of view $[\mathrm{FOV}]=240 \times$ $240 \mathrm{~mm}^{2}$; matrix $=128 \times 128$, slice thickness $=4 \mathrm{~mm}, 200$ sets of 30 contiguous axial slices, parallel to the AC-PC plane). Total acquisition time of RS fMRI was approximately 10 minutes. During scanning, subjects were instructed to remain motionless, to keep their eyes closed, and not to think about anything in particular. The following structural MRI sequences were also acquired: (1) T2-weighted spin echo (SE) (TR = $3500 \mathrm{~ms}$, TE $=85$ $\mathrm{ms}$, echo train length $=15$, flip angle $=90^{\circ}, 22$ contiguous, 5 -mm-thick axial slices, FOV $=230 \times 184 \mathrm{~mm}^{2}$, matrix $=512 \times$ 512); (2) fluid-attenuated inversion recovery (FLAIR) (TR $=11000$ $\mathrm{ms}$, TE $=120 \mathrm{~ms}$, flip angle $=90^{\circ}, 22$ contiguous, 5-mm-thick axial slices, $\mathrm{FOV}=230 \times 131 \mathrm{~mm}^{2}$, matrix size $\left.=512 \times 512\right)$; (3) 3D T1-weighted fast field echo (TR $=25 \mathrm{~ms}$, TE $=4.6 \mathrm{~ms}$, flip angle $=30^{\circ}, 220$ contiguous axial slices with voxel size $=0.89 \times$ $0.89 \times 0.8 \mathrm{~mm}, \mathrm{FOV}=230 \times 182 \mathrm{~mm}^{2}$, matrix size $\left.=256 \times 256\right)$; and (4) pulsed-gradient SE EP with sensitivity encoding (acceleration factor $=2.5$, TR $=8986 \mathrm{~ms}, \mathrm{TE}=80 \mathrm{~ms}, 55$ contiguous, 2.5-mm-thick axial slices, number of acquisitions $=2$; after SENSE reconstruction, the matrix dimension of each slice was $256 \times 256$, with an in-plane pixel size of $0.94 \times 0.94 \mathrm{~mm}$ and a FOV of $240 \times 240 \mathrm{~mm}^{2}$ ) and with diffusion gradients applied in 32 non-collinear directions, using a gradient scheme that is standard on this system (gradient over-plus) and optimized to reduce echo time as much as possible. The $b$ factor used was 1000 $\mathrm{s} / \mathrm{mm}^{2}$. Fat saturation was performed to avoid chemical shift artifacts. All slices were positioned to run parallel to a line that joins the most infero-anterior and infero-posterior parts of the corpus callosum. 


\subsection{MRI analysis}

MRI analysis was performed by an experienced observer who was blinded to subjects' identity. WM hyperintensities, if any, were identified on T2-weighted and FLAIR scans. WMH load was measured using the Jim software package (version 5.0; Xinapse Systems, Northants, UK, http://www.xinapse.com).

\subsubsection{RS functional connectivity}

RS fMRI data analysis was carried out using MELODIC (Multivariate Exploratory Linear Optimized Decomposition into Independent Components) of FMRIB software library (FSL version 4.1.7, http://www.fmrib.ox.ac.uk/fsl/melodic/) (Beckmann et al., 2005). After having removed the first 4 volumes of each image to allow magnet signal stabilization, individual pre-statistical processing consisted of motion correction (http://www.fmrib.ox.ac.uk/ analysis/techrep/tr04ss2/tr04ss2/node13.html), removal of nonbrain tissue, spatial smoothing using a 6-mm full-width-at-halfmaximum Gaussian kernel, and high-pass temporal filtering equivalent to 100 seconds $(0.01 \mathrm{~Hz})$. None of the study participants were excluded from analysis because of motion, as the maximum cumulative translation was $<1.5 \mathrm{~mm}$ and the maximum rotation was $<0.3$ degrees for all participants. fMRI volumes were then registered to the individual's 3-dimensional T1-weighted scan and standard space images using FMRIB's Nonlinear Image Registration Tool (FNIRT). Pre-processed fMRI data, containing 196 time-points for each subject, were temporally concatenated across subjects to create a single 4-dimensional dataset. This fMRI dataset was then decomposed into independent components (IC) with a free estimation for the number of components.

\subsubsection{DT MRI preprocessing}

DT MRI analysis was performed using FSL tools (FSL version 4.1.7, http://www.fmrib.ox.ac.uk/fsl/fdt/index.html) and the JIM5 software. Briefly, the diffusion-weighted data were skull-stripped using the Brain Extraction Tool implemented in FSL. Then, using FMRIB's Linear Image Registration Tool (FLIRT), the 2 acquired diffusionweighted scans were coregistered by applying the rigid transformation needed to correct for position between the $2 b_{0}$ images (T2-weighted, but not diffusion-weighted), and a rotation component was also applied to diffusion-weighted directions. Eddy current correction was then performed using the JIM5 software, and the 2 acquisitions were concatenated. Finally, the DT was estimated on a voxel-by-voxel basis using DTIfit provided by the FMRIB Diffusion Toolbox; and maps of fractional anisotropy (FA) were obtained.

\subsubsection{Tractography: seed definition}

Seeds for tractography of the CST, corpus callosum, and superior longitudinal fasciculus (SLF) were defined in the Montreal Neurological Institute (MNI) space on the FA template provided by FSL. Regions of interest (ROI) were defined manually on sagittal or axial slices based on a priori knowledge of the anatomy of the tracts. Seeds were drawn where these tracts pass through a bottleneck to include the highest number of fibers constituting the tract in the starting seed. Tractography was then performed using a single-seed approach. Masks were used to exclude fibers from neighboring tracts. For the CST, an axial ROI was drawn at the top of the bulbar pyramids of each side and included 4 slices. The seed for the corpus callosum was a sagittal ROI that included the 4 median slices on which the corpus callosum was clearly visible. For the SLF, a single coronal seed was drawn on 3 coronal slices posterior to the postcentral gyrus, in the vicinity of the supramarginal gyrus of each side.
The corpus callosum was also partitioned following 2 procedures. First, we divided the sagittal seed ROI used to obtain the entire corpus callosum into 3 parts along its anteroposterior axis, which roughly correspond to the genu, body, and splenium. Second, the corpus callosum was segmented into 3 portions to identify the callosal fibers linking the PMC, lateral premotor cortices, and supplementary motor area (SMA). To this end, the same seed and exclusion masks of the entire corpus callosum were used, and, in addition, each of the following 3 cortical areas were used as target ROI: PMC, corresponding to the bilateral precentral gyrus as defined by the Harvard-Oxford cortical atlas (http://www.cma.mgh.harvard.edu); SMA, corresponding to the bilateral supplementary motor cortex as defined by the HarvardOxford cortical atlas; and the premotor cortex, which was drawn manually and included the dorsolateral part of Brodmann area 6 (from MNI y $=5$ to MNI y $=29$ ). This ROI corresponds to the posterior parts of the middle and inferior frontal gyri on the Harvard-Oxford cortical atlas.

The seeds, targets, and exclusion masks were first transformed to each subject's native diffusion space, using the inverse of the linear and nonlinear transformations obtained previously to align each subject's FA to the standard space, and then binarized. Transformed seeds, targets, and exclusion masks were evaluated visually by an experienced observer.

\subsubsection{Tractography: fiber tracking}

Fiber tracking was performed in native DT MRI space using a probabilistic tractography algorithm implemented in FSL (probtrackx), which is based on a Bayesian estimation of diffusion parameters (Bedpostx) (Behrens et al., 2007). Fiber tracking was initiated from all voxels within the seed masks in the diffusion space to generate 5000 streamline samples, with a step length of $0.5 \mathrm{~mm}$ and a curvature threshold of 0.2. CST and SLF were run separately for the right and left hemispheres. Tract maps were then normalized, taking into consideration the numbers of voxels in the seed masks. To do so, the number of streamline samples present in the voxels of the tract maps was divided by the waytotal, which corresponds to the total number of streamline samples that were not rejected by the exclusion masks (http://fsl. fmrib.ox.ac.uk/fsl/fslwiki/FDT/UserGuide) (Behrens, et al., 2007). The tract masks obtained were thresholded at a value equal to $40 \%$ of the $95^{\text {th }}$ percentile of the distribution of the intensity values in the voxels included in the tracts (Galantucci et al., 2011). This normalization procedure allowed us to correct for possible differences between tracts due to the different sizes of the starting seeds. In this way, we also excluded background noise and avoided a too-restrictive thresholding when the maximum intensity value was an outlier. Using a "single-seed" approach, the reconstruction of the corpus callosum and bilateral CST and SLF was obtained. Using a "seed-to-target" approach, the partitions of the corpus callosum (namely CC-genu, CC-body, CC-splenium, CC-PMC, CC-premotor, and CC-SMA) were produced. For each tract, the average FA was calculated in the native space.

Group probability maps of each tract were produced to visually check the anatomical accuracy of the tracts of interest across the study subjects. Individual tracts were transformed to MNI space and averaged across subjects. Only voxels present in at least $10 \%$ of the subjects were shown (Fig. 1).

\subsection{Statistical analysis}

\subsubsection{Demographic, clinical, cognitive, and structural MRI data}

Group differences in categorial variables were assessed using the Fisher exact test. Continuous variables were compared using 


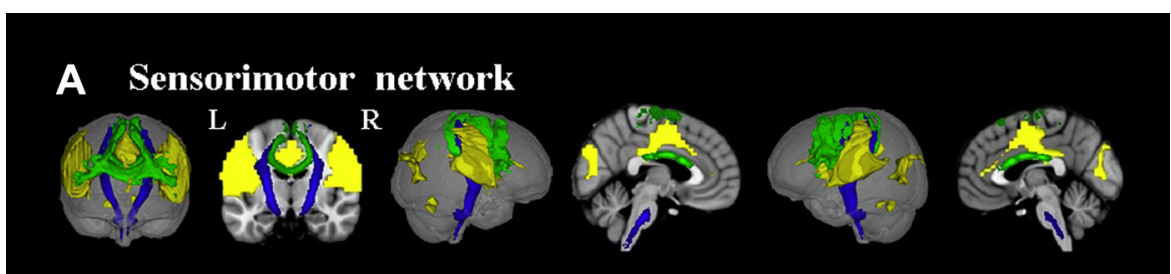

\section{B Default mode network}

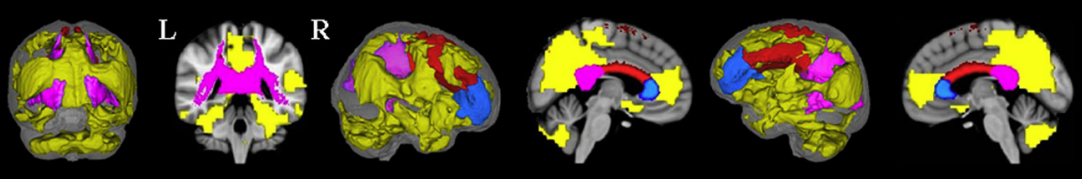

\section{Frontal network}
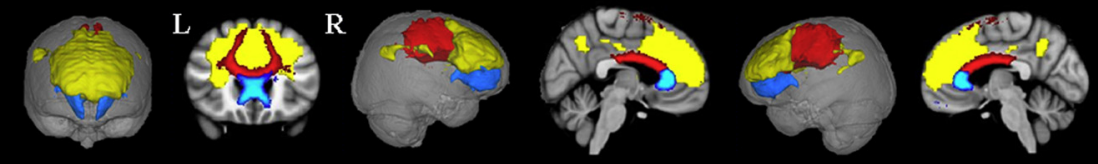

\section{Left frontoparietal network}
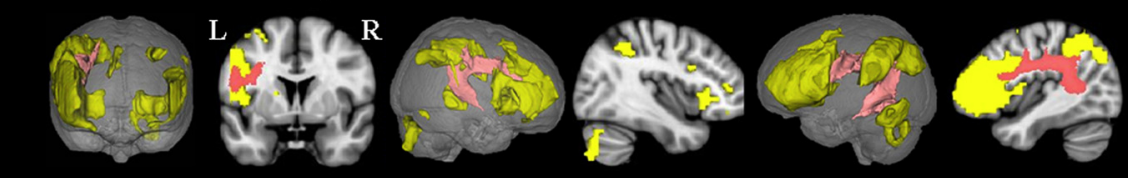

\section{E Right frontoparietal network}

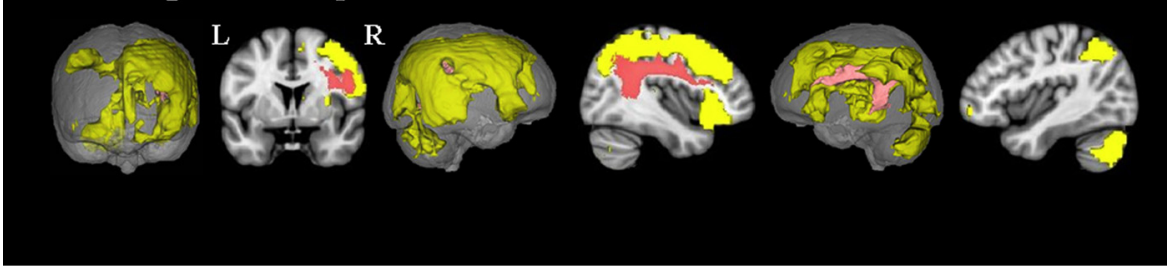

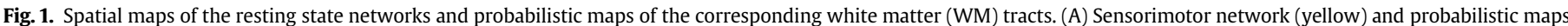

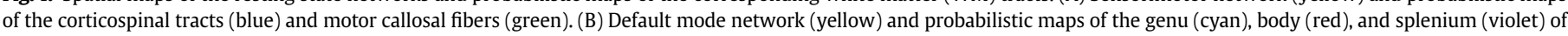

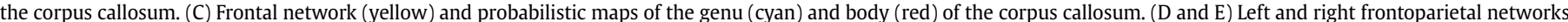

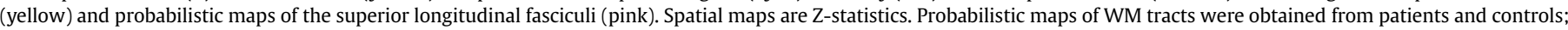

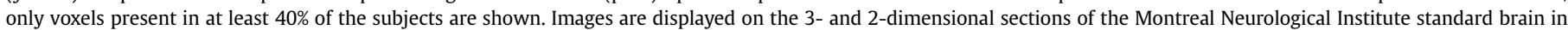
neurological convention.

the Mann-Whitney $U$ test (SAS release 9.1; SAS Institute, Cary, NC) $(p<0.05)$.

\subsubsection{RS functional connectivity: between-group comparisons}

Between-subjects analysis of the RS fMRI data was carried out using a novel dual-regression technique (Filippini et al., 2009), an approach that allows to identify subject-specific temporal dynamics and spatial maps that are associated with each group IC map. Among group-IC spatial maps, IC of interest (sensorimotor network, DMN, frontal network, and left and right frontoparietal networks) were selected by visual inspection based on previous literature (Beckmann et al., 2005; Damoiseaux et al., 2006; Smith et al., 2009) and the frequency spectra of the time courses of the components (i.e., more than $90 \%$ of spectral power less than $0.08 \mathrm{~Hz}$ ). Then, the dualregression procedure involves the following: the use of selected group-IC spatial maps in a linear model fit (spatial regression) against the single-subject fMRI data sets, resulting in matrices describing temporal dynamics for each IC and subject; and the use of these time-course matrices in a linear model fit (temporal regression) against the associated fMRI data set to estimate subject-specific spatial maps (Filippini et al., 2009). After the dual regression, spatial maps of all subjects were collected into single 4-dimensional files for each original IC. Nonparametric permutation tests (5000 permutations) were used to detect statistically significant differences between groups within the RSN of interest obtained with MELODIC (the single 4-dimensional files for each original IC) (Nichols and Holmes, 2002). The analyses were adjusted for age. Furthermore, analyses were restricted within the spatial RSN of interest using binary masks obtained by thresholding the corresponding $\mathrm{Z}$ map image $(Z>2.3)$. A family-wise error (FWE) correction for multiple comparisons was performed, implementing threshold-free cluster enhancement (TFCE) using a significance threshold of $p<0.05$ and a cluster extent $>20$ (Smith and Nichols, 2009).

\subsubsection{RS functional connectivity: correlations with clinical,} cognitive, and DT MRI data

Correlations analyses between functional connectivity maps, clinical and cognitive scores, and FA values of WM tracts were 
performed. To minimize the number of comparisons, we defined a priori the variables to be correlated with each RSN (Table 2) on the basis of functional and anatomical knowledge. Based on the results of the between-group comparisons, correlations with clinical, cognitive, and DT MRI variables were then limited to those clusters that showed significant group differences. A TFCE-corrected, permutation-based, non-parametric testing was used to assess such correlations $(p<0.05$; cluster extent $>20)$. Results that did not survive the correction for multiple comparisons were reported at an uncorrected threshold ( $p<0.05$; cluster extent $>20)$.

\section{Results}

\subsection{Demographic, clinical, cognitive, and structural MRI data}

PLS patients and healthy controls did not differ in terms of age, sex, and WM lesion load (Table 1). The neuropsychological features of PLS patients are shown in Table 3. In agreement with the Strong consensus criteria (Strong et al., 2009), 2 PLS patients were found to be cognitively impaired. In addition, 1 PLS patient had abnormal scores in 1 executive and 1 non-executive function test (language), whereas 1 PLS patient showed abnormal scores in 1 executive test only and 4 patients in 1 non-executive test only (3 language, and 1 memory). PLS patients compared with controls showed reduced FA values of the CST bilaterally and the CC-PMC and CC-SMA tracts (Table 1).

\subsection{RS functional connectivity: components}

The group IC analysis estimated 34 components. Based on their frequency spectra, spatial patterns, and previous literature (Beckmann et al., 2005; Damoiseaux et al., 2006; Smith et al., 2009), the sensorimotor network, DMN, frontal network, and right and left frontoparietal networks were identified (Fig. 1).

\subsection{RS functional connectivity: between-group comparisons}

\subsubsection{Sensorimotor network}

Compared with controls, PLS patients showed an increased functional connectivity of the precentral and postcentral gyri, bilaterally ( $p<0.05$, FWE-corrected; Table 4, Fig. 2A). No region of decreased functional connectivity was found in PLS patients relative to controls.

\subsubsection{Frontal network}

In PLS patients compared with controls, we observed an increased functional connectivity of the anterior cingulate cortex (ACC) and superior medial frontal gyrus bilaterally, left SMA, and right insula ( $p<0.05$, FWE-corrected; Table 4, Fig. 2B). No region of decreased functional connectivity was found in PLS patients relative to controls.

\subsubsection{Left frontoparietal network}

PLS patients relative to controls showed an increased functional connectivity of the left middle orbitofrontal, inferior frontal, and superior temporal gyri ( $p<0.05$, FWE-corrected; Table 4, Fig. 2C). No region of decreased functional connectivity was found in PLS patients relative to controls.

\subsubsection{DMN and right frontoparietal network}

No functional connectivity difference was found in the DMN and right frontoparietal network between patients and controls.

\subsection{Correlations}

\subsubsection{Sensorimotor network}

Sensorimotor network results are shown in Table 5. In the sensorimotor network, the increased functional connectivity of the right pre- and postcentral gyri was associated with lower ALSFRS-r scores ( $p<0.05$, uncorrected). The increased functional connectivity of the right postcentral gyrus was also related to a more rapid disease progression rate $(p<0.05$, uncorrected). No correlation was found between the sensorimotor network functional connectivity and the disease duration and UMN score. In the sensorimotor network, the increased functional connectivity of the left pre- and postcentral gyri correlated with reduced FA values of the CC-PMC, whereas the increased functional connectivity of the right precentral gyrus was associated with reduced FA values of the CC-SMA ( $p<0.05$, uncorrected).

\subsubsection{Frontal network}

Frontal network results also are shown in Table 5. In the frontal network, the increased functional connectivity of the right superior frontal gyrus was associated with a better performance at the WCST global score; whereas the increased functional connectivity of the left superior frontal gyrus and right insula was associated with a worse performance at the phonemic and semantic fluency tests, respectively ( $p<0.05$, uncorrected). The increased functional connectivity of the left SMA and right superior frontal gyrus and insula was associated with reduced FA values of the CC-body ( $p<0.05$, uncorrected). No correlation was found between the frontal network functional connectivity and the FA values of the CC-genu.

\subsubsection{Left frontoparietal network}

No relationship was found between the functional connectivity of left frontoparietal network, cognitive variables, and DT MRI metrics.

Table 2

Correlation analysis: Clinical, cognitive, and DT MRI variables selected for each RSN of interest

\begin{tabular}{|c|c|c|c|c|}
\hline & Sensorimotor network & DMN & Frontal network & Left and right frontoparietal network \\
\hline Clinical and cognitive variables & $\begin{array}{l}\text { ALSFRS-r } \\
\text { UMN score } \\
\text { Progression rate } \\
\text { Disease duration }\end{array}$ & $\begin{array}{l}\text { MMSE } \\
\text { Rey's word list }\end{array}$ & $\begin{array}{l}\text { Phonemic fluency } \\
\text { Semantic fluency } \\
\text { WCST-global score }\end{array}$ & $\begin{array}{l}\text { Phonemic fluency } \\
\text { Semantic fluency }\end{array}$ \\
\hline WM tracts (FA values) & $\begin{array}{l}\text { L CST } \\
\text { R CST } \\
\text { CC-PMC } \\
\text { CC-premotor } \\
\text { CC-SMA }\end{array}$ & $\begin{array}{l}\text { CC-genu } \\
\text { CC-body } \\
\text { CC-splenium }\end{array}$ & $\begin{array}{l}\text { CC-genu } \\
\text { CC-body }\end{array}$ & $\begin{array}{l}\text { L SLF } \\
\text { R SLF }\end{array}$ \\
\hline
\end{tabular}

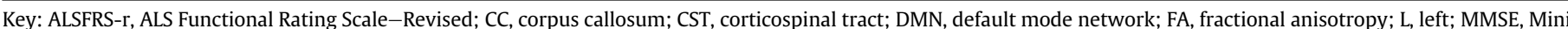

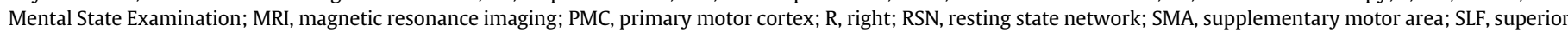
longitudinal fasciculus; WCST, Wisconsin Card Sorting test; WM, white matter. 
Table 3

Neuropsychological profile of PLS patients

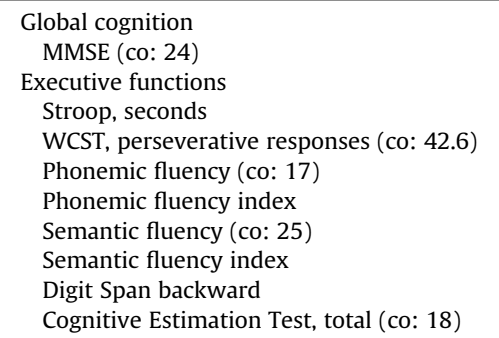

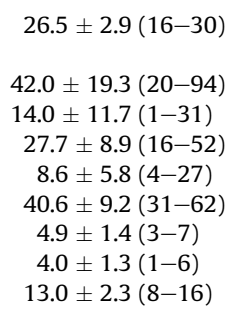

$\begin{array}{lc}\text { Reasoning and problem solving functions } & \\ \text { Raven's progressive matrices (co: 18) } & 29.5 \pm 4.7(18-35) \\ \text { Weigl test (co: 4.5) } & 12.4 \pm 1.8(9-16) \\ \text { WCST, global score (co: 90.5) } & 48.2 \pm 34.5(8-102) \\ \text { Memory } & 5.6 \pm 1.3(3-9) \\ \text { Digit span forward (co: 3.75) } & 41.7 \pm 9.3(31-62) \\ \text { Rey's word list, imm. recall (co: 28.53) } & 8.9 \pm 3.0(4-16) \\ \text { Rey's word list, delay recall (co: 4.69) } & \\ \text { Language } & 0.7 \pm 0.7(0-2) \\ \text { BADA, oral object naming, errors (co: 2) } & 1.8 \pm 1.5(0-5) \\ \text { BADA, oral action naming, errors (co: } 2) & \end{array}$

Reasoning and problem solving functions

WCST, global score (co: 90.5)

Digit span forward (co: 3.75$)$

Rey's word list, imm. recall (co: 28.53)

BADA, oral object naming, errors (co: 2)

$1.8 \pm 1.5(0-5)$

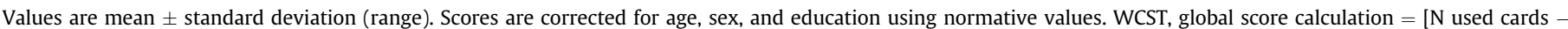

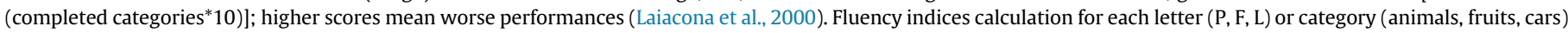

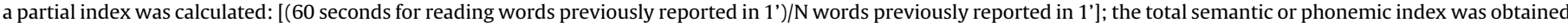

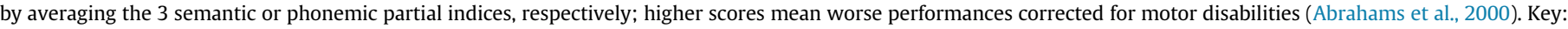
BADA, “Batteria per l'Analisi del Deficit Afasico"; co, cut-off; MMSE, Mini Mental State Examination; WCST, Wisconsin Card Sorting test.

\section{Discussion}

This RS fMRI study demonstrated increased functional connectivity in PLS spanning sensorimotor, medial, and dorsal frontal, insular, and superior temporal regions. Patients with more severe physical disability and more rapid rates of disease progression had increased connectivity values. The level of functional connectivity within the frontal network appeared to be related to executive dysfunction. In addition, the pattern of increased functional connectivity within the sensorimotor and frontal networks was associated with a greater structural damage to network-specific WM tracts. These findings suggest that an abnormal functional connectivity may be another factor characterizing the pathogenesis of PLS. We discuss these results in relation to the previous RS fMRI studies in ALS, taking into account the current literature on the physiopathology of PLS.

In ALS, initial RS fMRI studies confirmed the intuitive expectation of a decrease in functional connectivity in the sensorimotor network and DMN (Mohammadi et al., 2009; Tedeschi et al., 2010), supported by the evidence of an impaired interhemispheric functional connectivity between the PMC (JelsoneSwain et al., 2011). However, subsequent studies have identified regions of increased functional connectivity, including sensorimotor (Agosta et al. 2011; Douaud et al. 2011; Verstraete et al. 2010) and extra-motor (Agosta et al. 2013a; Douaud et al. 2011;
Luo et al. 2012) areas. A possible explanation of such a finding is that increased functional connectivity reflects a compensatory process in relation to the structural breakdown of the motor and frontotemporal systems. The relationship of decreased functional connectivity with clinical and cognitive status in 2 studies of ALS agree with this hypothesis (Agosta et al., 2011, 2013a). However, other studies of ALS found the most increased functional connectivity values in those patients with a faster disease progression (Douaud et al., 2011; Verstraete et al., 2010). In a study using a pre-defined ALS-specific cortical network, increased functional connectivity was found over a large area spanning sensorimotor, premotor, prefrontal, and thalamic regions, which significantly overlapped with areas of structural damage identified using DT MRI (Douaud et al., 2011). These observations lend support to a pathogenic loss of inhibitory neuronal influence on cortical activity, rather than only compensatory recruitment, to explain the increased functional connectivity in ALS (Turner and Kiernan, 2012).

The hypothesis of a central interneuronopathy as a key aspect of ALS pathogenesis has been previously postulated using histopathology, neurophysiological techniques, and positron emission tomography (PET) (Bae et al., 2013; Turner and Kiernan, 2012). In ALS, post mortem studies showed loss of interneuron-specific calcium-binding proteins (Maekawa et al., 2004) and $\gamma$-amino butyric acid (GABA) receptor mRNA expression (Petri et al., 2003).

Table 4

Regions of increased functional connectivity of the sensorimotor, frontal and left frontoparietal networks in PLS patients compared with controls*

\begin{tabular}{|c|c|c|c|c|c|}
\hline \multicolumn{6}{|c|}{ Sensorimotor network } \\
\hline No. of voxels & Region & $\mathrm{x}$ & $\mathrm{y}$ & $\mathrm{z}$ & $\mathrm{Z}$ \\
\hline \multirow[t]{3}{*}{67} & $\mathrm{R}$ precentral gyrus & 58 & -8 & 42 & 0.989 \\
\hline & $\mathrm{R}$ postcentral gyrus & 62 & -10 & 40 & 0.987 \\
\hline & & 50 & -10 & 36 & 0.981 \\
\hline \multirow[t]{3}{*}{38} & L postcentral gyrus & -50 & -10 & 30 & 0.967 \\
\hline & L precentral gyrus & -54 & -6 & 34 & 0.959 \\
\hline & L postcentral gyrus & -46 & -14 & 36 & 0.957 \\
\hline \multicolumn{6}{|c|}{ Frontal network } \\
\hline 472 & L superior medial frontal gyrus & -10 & 64 & 10 & 0.985 \\
\hline 434 & $\mathrm{R}$ insula & 34 & 22 & 12 & 0.987 \\
\hline 233 & $\mathrm{R}$ anterior cingulate cortex & 6 & 26 & 18 & 0.979 \\
\hline 76 & $\mathrm{~L}$ anterior cingulate cortex & -8 & 42 & 12 & 0.961 \\
\hline 59 & R superior medial frontal gyrus & 10 & 46 & 40 & 0.970 \\
\hline 21 & L SMA & -6 & 16 & 60 & 0.964 \\
\hline \multicolumn{6}{|c|}{ Left frontoparietal network } \\
\hline 122 & L middle orbitofrontal gyrus & -36 & 48 & -6 & 0.987 \\
\hline 43 & L inferior frontal gyrus & -50 & 40 & 16 & 0.987 \\
\hline 42 & L superior temporal gyrus & -54 & -34 & 18 & 0.989 \\
\hline
\end{tabular}

The letters $\mathrm{x}, \mathrm{y}$, and $\mathrm{z}$ are coordinates in the Montreal Neurological Institute space. Key: L, left; R, right; SMA, supplementary motor area.

$* p<0.05$, family-wise error-corrected. 


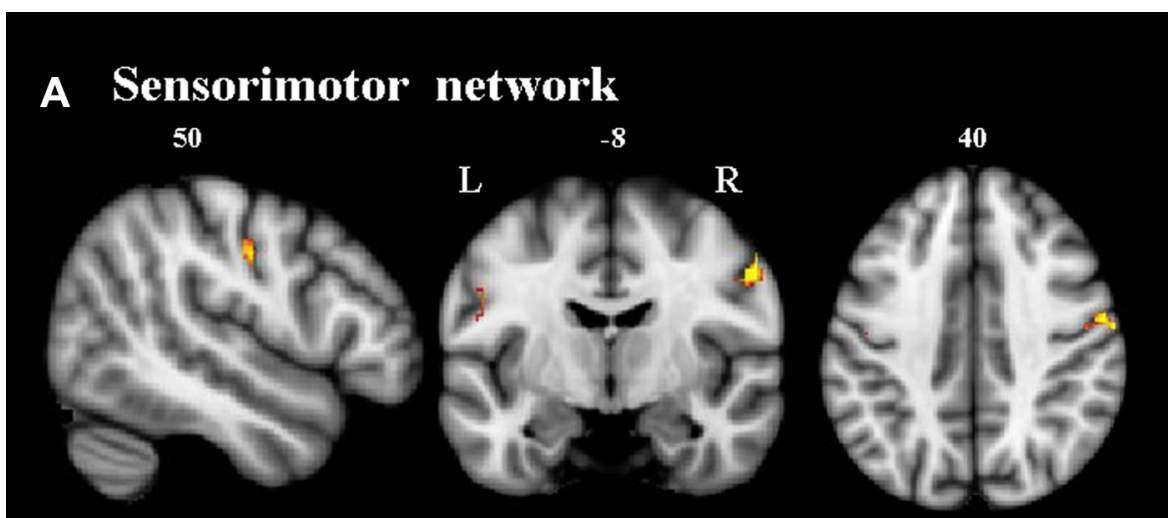

\section{B Frontal network}
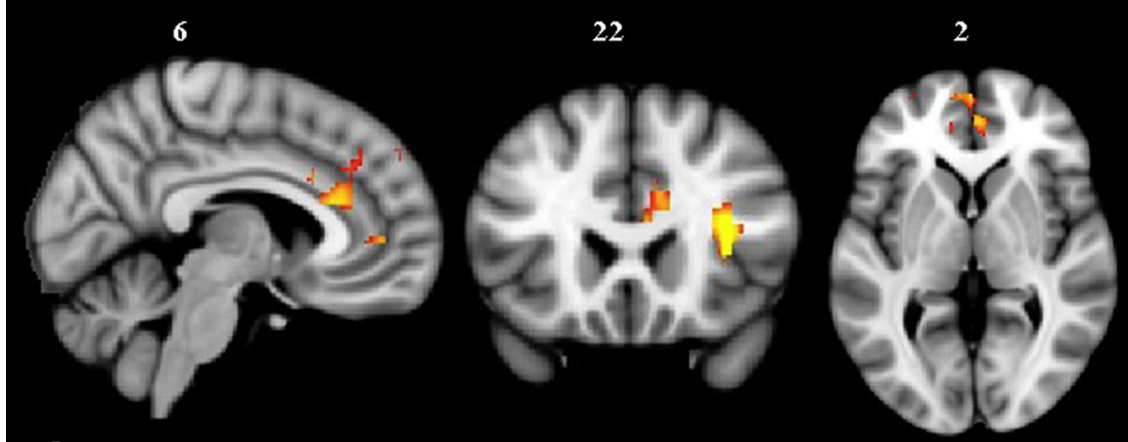

\section{Left frontoparietal network}

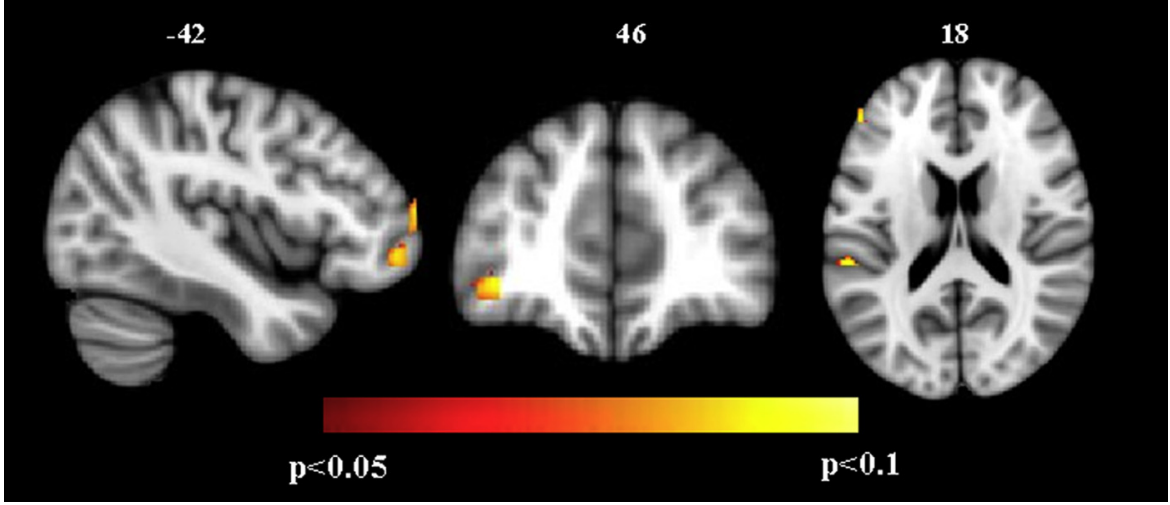

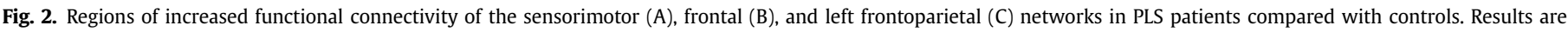
shown at $p<0.05$, family-wise error corrected, on the Montreal Neurological Institute standard brain in neurological convention.

Transcranial magnetic stimulation studies of ALS patients showed a deficient transcallosal inhibition and increased cortical excitability (Riva, et al., 2012; Wittstock et al., 2007). Cortical hyperexcitability was found to precede the development of clinical symptoms in presymptomatic SOD1 mutation carriers (Vucic et al., 2008). In ALS, PET detected a distributed loss of flumazenil binding (Lloyd et al., 2000), a benzodiazepine receptor antagonist that can be used to visualize the distribution of $\mathrm{GABA}_{\mathrm{A}}$ receptors in the human brain. Furthermore, investigators in a recent proton magnetic resonance spectroscopy study observed that, compared with healthy controls, patients with ALS had lower levels of GABA in the left motor cortex (Foerster et al., 2012). Little is known about inhibitory circuitry involvement in PLS except that PET flumazenil binding is reduced in the motor cortex, ACC, and parietal and temporal lobes of these patients (Turner et al., 2007).
The positive relationship of sensorimotor functional connectivity with disease severity and rate of disease progression, and the DT MRI data observed in our study, support the notion that an increased functional connectivity is likely to have a role in the pathogenesis of PLS. Indeed, we found a correlation between increased sensorimotor functional connectivity and damage to the transcallosal motor fibers. In addition to CST involvement, PLS patients also showed a marked damage to the motor fibers of the corpus callosum, in keeping with pathological reports (Konagaya et al., 1998; Tan et al., 2003) and previous DT MRI studies (Ciccarelli et al., 2009; Iwata et al., 2011; Muller et al., 2012; Unrath et al., 2010; van der Graaff et al., 2011). Since callosal neurons are excitatory and their major targets are the inhibitory interneurons of the contralateral hemisphere (Li and Pleasure, 2011), our findings are in accordance with the interneuronopathy theory suggesting that 
Table 5

Correlation analysis: relationships of sensorimotor and frontal network functional connectivity with clinical, cognitive, and DT MRI variables*

\begin{tabular}{|c|c|c|c|c|c|}
\hline \multicolumn{6}{|c|}{ Sensorimotor network } \\
\hline No. of voxels & Region & $\mathrm{x}$ & $\mathrm{y}$ & $\mathrm{z}$ & Z \\
\hline \multicolumn{6}{|c|}{ ALSFRS-r (negative relationship) } \\
\hline \multirow[t]{3}{*}{87} & R precentral gyrus & 58 & 6 & 36 & 0.993 \\
\hline & & 54 & -2 & 42 & 0.977 \\
\hline & & 62 & 8 & 26 & 0.951 \\
\hline 35 & R postcentral gyrus & 66 & -12 & 36 & 0.988 \\
\hline \multicolumn{6}{|c|}{ Disease progression rate (positive relationship) } \\
\hline 192 & R postcentral gyrus & 70 & -12 & 32 & 0.991 \\
\hline \multicolumn{6}{|c|}{ CC-PMC FA (negative relationship) } \\
\hline \multirow[t]{2}{*}{54} & L postcentral gyrus & -62 & 4 & 14 & 0.984 \\
\hline & L precentral gyrus & -52 & 8 & 16 & 0.971 \\
\hline 29 & L postcentral gyrus & -58 & -6 & 48 & 0.976 \\
\hline \multicolumn{6}{|c|}{ CC-SMA FA (negative relationship) } \\
\hline 26 & $\mathrm{R}$ precentral gyrus & 58 & 8 & 38 & 0.975 \\
\hline \multicolumn{6}{|c|}{ Frontal network } \\
\hline \multicolumn{6}{|c|}{ Phonemic fluency (negative relationship) } \\
\hline 33 & L superior frontal gyrus & -20 & 14 & 54 & 0.982 \\
\hline \multicolumn{6}{|c|}{ Semantic fluency (negative relationship) } \\
\hline 45 & $\mathrm{R}$ insula & 30 & 26 & 8 & 0.982 \\
\hline \multicolumn{6}{|c|}{ WCST global score (negative relationship) } \\
\hline 241 & R superior frontal gyrus & 18 & 34 & 32 & 0.991 \\
\hline \multicolumn{6}{|c|}{ CC-body FA (negative relationship) } \\
\hline 332 & $\mathrm{R}$ superior frontal gyrus & 18 & 38 & 48 & 0.991 \\
\hline 74 & $\mathrm{R}$ insula & 34 & 22 & 12 & 0.968 \\
\hline 31 & L SMA & -6 & 10 & 62 & 0.991 \\
\hline
\end{tabular}

The letters $\mathrm{x}, \mathrm{y}$, and $\mathrm{z}$ are coordinates in the Montreal Neurological Institute space. Key: ALSFRS-r, ALS Functional Rating Scale-Revised; CC, corpus callosum; CST, corticospinal tract; FA, fractional anisotropy; L, left; MRI, magnetic resonance imaging; PMC, primary motor cortex; R, right; SMA, supplementary motor area; WCST, Wisconsin Card Sorting test.

${ }^{*} p<0.05$ uncorrected.

severe microstructural damage to the corpus callosum of PLS patients contributes to their altered cortical excitability.

PLS patients showed an increased functional connectivity compared with controls not only in the sensorimotor areas but also in the frontal and left frontoparietal networks, supporting the concept that PLS, like ALS, is a multisystem disorder. Frontal and frontoparietal networks sustain executive functions, problem solving, attentional processes, and emotional salience processing, and overlap largely with regions recruited during the performance of cognitively demanding tasks (Smith et al., 2009). Although PLS seems to hit mainly the PMC, diseaserelated degeneration with ubiquitin inclusions is known to extend anteriorly to the non-motor frontal cortices and ventrally to the temporal cortices (Konagaya et al., 1998; Kosaka et al., 2011; Mochizuki et al., 2004; Tan et al., 2003). Phosphorylated TDP-43 immunohistochemistry in PLS revealed the presence of many positively stained neuronal cytoplasmic inclusions and dystrophic neuritis/neuropil threads in the affected frontotemporal cortex and subcortical nuclei (Kosaka et al., 2011). DT MRI study of PLS patients found WM abnormalities in extramotor areas (Agosta et al., 2013b; Iwata et al., 2011; van der Graaff et al., 2011). Cognition has received much lower attention in PLS than ALS. In early studies, cognition was reported to be normal in PLS (Konagaya et al., 1998; Tan et al., 2003). However, other investigators have reported a frontal lobe dysfunction syndrome in PLS cases (Caselli et al. 1995; Le Forestier et al., 2001; Piquard et al., 2006; Zago et al., 2008). A prospective study (Grace et al., 2005) found similar proportions of PLS and ALS patients who had deficits of verbal fluency and memory. In our study, roughly $15 \%$ of PLS patients showed frontal lobe dysfunction characterized by deficits in set-shifting and/or verbal fluency. The lower proportion of PLS patients with cognitive impairment in our cohort compared with previous ones (Grace et al., 2005; Piquard et al., 2006) may result from patient selection and different criteria used to define cognitive impairment. In addition, although the MRI protocol did not require active tasks, our patients should have been able to lie quietly in the scanner for about 1 hour. We believe that this may have selected higher-functioning subjects relative to those in previous studies that did not use strict inclusion/exclusion criteria.

An altered recruitment of the prefrontal cortex has been previously observed in ALS patients during letter fluency and confrontation naming tasks (Abrahams et al., 1996, 2004). Therefore, a disorganization of the frontal networks may contribute to the appearance of cognitive impairment also in PLS. However, the interpretation of the correlations that we found among frontal functional connectivity, cognitive variables, and WM tract damage is challenging. The functional connectivity of the left frontal and right insular regions of PLS patients was higher, and their verbal fluency deficits and damage to the CCbody were more severe. Conversely, the increased functional connectivity of the right superior frontal gyrus was associated with a better performance at the WCST test. These findings are counterintuitive, as the increased functional connectivity of the frontal cortex could be interpreted as a pathogenetic factor in the first case (as described above for the sensorimotor areas) and as a physiological compensation in an attempt to maintain an efficient cognitive performance in the second case. A possible explanation of such a complex constellation of findings could be a more heterogeneous spatial and temporal progression of cortical degeneration in extramotor compared with motor regions in MND patients (Bae et al., 2013). Longitudinal studies assessing the evolution of cortical involvement, WM tract damage, and cognitive deficits in PLS are now warranted to clarify this issue.

Previous studies investigating DMN connectivity in ALS reported conflicting results, with some authors detecting a reduced DMN connectivity in both the ACC and medial prefrontal cortices and the posterior cingulate and inferior parietal cortices (Mohammadi et al., 2009), and others finding a divergent connectivity pattern with a decreased connectivity of the frontal cortex and an increased connectivity of the precuneus (Agosta et al., 2013a), or no abnormalities (Tedeschi et al., 2010). Discordance across studies may be due to methodological differences in the fMRI analysis or inclusion of patients with different clinical and cognitive features. The low prevalence of cognitive deficits in our study may explain the DMN integrity in PLS patients.

A possible limitation of our study is that, as in the majority of previous reports, PLS was diagnosed according to the criteria of Pringle et al. (Pringle et al., 1992), which have a low specificity, as they do not allow exclusion of patients with subtle EMG abnormalities. Nevertheless, although we selected patients using the criterion of minimum 3 years from symptom onset (Pringle et al., 1992), only 2 subjects had a disease duration of less than 48 months at the time of MRI scan ( 38 and 46 months, respectively). Both patients have now been followed up clinically and with EMG for at least 1 additional year and did not develop LMN signs. In addition, correlation results did not survive the correction for multiple comparisons. However, the a priori definition of the variables to be correlated with each RSN of interest, as well as the fact that correlation analysis was limited to those clusters that showed significant group differences, should have minimized false-positive results. Finally, the objective of the present study was to apply a multimodal imaging approach to PLS patients to explore RSN characteristics in relation to clinical and cognitive features. Future studies should compare functional and structural connectivity in early PLS and ALS patients to identify diagnostic and prognostic markers in these conditions. 


\section{Disclosure statement}

F.A. serves on the editorial board of the Journal of Neurology; and has received research support from the Italian Ministry of Health, funding for travel from Teva Pharmaceutical Industries Ltd, and speaker honoraria from Bayer Schering Pharma, Biogen Idec, Sanofi Aventis, and Serono Symposia International Foundation. E.C., A.I., S.M., and A.Fal. report no conflicts of interest. A.C. serves on a scientific advisory board for Biogen Idec and Cytokinetics; serves on the editorial advisory board of Amyotrophic Lateral Sclerosis; and has received research support from Italian Ministry of Health, Regione Piemonte, University of Torino, Federazione Italiana Giuoco Calcio. The research leading to these results has received funding from the European Community's Health Seventh Framework Programme (FP7/2007-2013) (grant agreements no. 259867 and 278611). N.R. has received research support from Agenzia di Ricerca per la Sclerosi Laterale Amiotrofica (AriSLA). V.S. has received research support from Agenzia di Ricerca per la Sclerosi Laterale Amiotrofica (AriSLA) cofinanced with support of " $5 \times 1000$ " - Healthcare Research of the Ministry of Health (EXOMEFALS). A.C. has received research support from Regione Piemonte, Compagnia di San Paolo and Italian Ministry of Health. G.C. has received compensation for consulting services and/or speaking activities from Novartis, Teva Pharmaceutical Ind., Sanofi, Genzyme, Merck Serono, Biogen Bayer, Actelion and Serono Symposia International Foundation. M.F. serves on scientific advisory boards for Teva Pharmaceutical Industries Ltd and Genmab A/S; has received compensation for consulting services and/or speaking activities from Bayer Schering Pharma, Biogen Idec, Genmab A/S, Merck Serono, and Teva Pharmaceutical Industries Ltd; receives research support from Bayer Schering Pharma, Biogen Idec, Genmab A/S, Merck Serono, Novartis, Teva Pharmaceutical Industries Ltd, Fondazione Italiana Sclerosi Multipla, the Italian Ministry of Health, CurePSP, and the Gossweiler Foundation (Switzerland).

\section{Acknowledgements}

This study was partially supported by a grant from the Italian Ministry of Health (grant RF-2010-2313220) and the European Community's Health Seventh Framework Programme (FP7/ 2007-2013) (grant agreement 259867).

\section{References}

Abrahams, S., Goldstein, L.H., Kew, J.J., Brooks, D.J., Lloyd, C.M., Frith, C.D., Leigh, P.N., 1996. Frontal lobe dysfunction in amyotrophic lateral sclerosis. A PET study. Brain 119, 2105-2120.

Abrahams, S., Goldstein, L.H., Simmons, A., Brammer, M., Williams, S.C., Giampietro, V., Leigh, P.N., 2004. Word retrieval in amyotrophic lateral sclerosis: a functional magnetic resonance imaging study. Brain 127, 1507-1517.

Abrahams, S., Leigh, P.N., Harvey, A., Vythelingum, G.N., Grise, D., Goldstein, L.H., 2000. Verbal fluency and executive dysfunction in amyotrophic lateral sclerosis (ALS). Neuropsychologia 38, 734-747.

Agosta, F., Canu, E., Valsasina, P., Riva, N., Prelle, A., Comi, G., Filippi, M., 2013a. Divergent brain network connectivity in amyotrophic lateral sclerosis. Neurobiol. Aging 34, 419-427.

Agosta, F., Galantucci, S., Riva, N., Chio, A., Messina, S., Iannaccone, S., Calvo, A., Silani, V., Copetti, M., Falini, A., Comi, G., Filippi, M., 2013b. Intrahemispheric and interhemispheric structural network abnormalities in PLS and ALS. Hum. Brain Mapp. In press. Epub ahead of print 30 April 2013; http://dx.doi.org/10.1002/ hbm.22286.

Agosta, F., Valsasina, P., Absinta, M., Riva, N., Sala, S., Prelle, A., Copetti, M., Comola, M., Comi, G., Filippi, M., 2011. Sensorimotor functional connectivity changes in amyotrophic lateral sclerosis. Cereb. Cortex 21, 2291-2298.

Bae, J.S., Simon, N.G., Menon, P., Vucic, S., Kiernan, M.C., 2013. The puzzling case of hyperexcitability in amyotrophic lateral sclerosis. J. Clin. Neurol. 9, 65-74.

Basso, A., Capitani, E., Laiacona, M., 1987. Raven's coloured progressive matrices: normative values on 305 adult normal controls. Funct. Neurol. 2, 189-194.
Beckmann, C.F., DeLuca, M., Devlin, J.T., Smith, S.M., 2005. Investigations into resting-state connectivity using independent component analysis. Philos. Trans. R. Soc. Lond. B Biol. Sci. 360, 1001-1013.

Behrens, T.E., Berg, H.J., Jbabdi, S., Rushworth, M.F., Woolrich, M.W., 2007. Probabilistic diffusion tractography with multiple fibre orientations: what can we gain? Neuroimage 34, 144-155.

Carlesimo, G.A., Caltagirone, C., Gainotti, G., 1996. The Mental Deterioration Battery: normative data, diagnostic reliability and qualitative analyses of cognitive impairment. The Group for the Standardization of the Mental Deterioration Battery. Eur. Neurol. 36, 378-384.

Caselli, R.J., Smith, B.E., Osborne, D., 1995. Primary lateral sclerosis: a neuropsychological study. Neurology 45, 2005-2009.

Cedarbaum, J.M., Stambler, N., Malta, E., Fuller, C., Hilt, D., Thurmond, B., Nakanishi, A., 1999. The ALSFRS-R: a revised ALS functional rating scale that incorporates assessments of respiratory function. BDNF ALS Study Group (Phase III). J. Neurol. Sci. 169, 13-21.

Chio, A., Calvo, A., Moglia, C., Mazzini, L., Mora, G., 2011. Phenotypic heterogeneity of amyotrophic lateral sclerosis: a population based study. J. Neurol. Neurosurg. Psychiatry 82, 740-746.

Ciccarelli, O., Behrens, T.E., Johansen-Berg, H., Talbot, K., Orrell, R.W., Howard, R.S. Nunes, R.G., Miller, D.H., Matthews, P.M., Thompson, A.J., Smith, S.M., 2009 Investigation of white matter pathology in ALS and PLS using tract-based spatial statistics. Hum. Brain Mapp. 30, 615-624.

Damoiseaux, J.S., Rombouts, S.A., Barkhof, F., Scheltens, P., Stam, C.J., Smith, S.M., Beckmann, C.F., 2006. Consistent resting-state networks across healthy subjects. Proc. Natl. Acad. Sci. U.S.A 103, 13848-13853.

Della Sala, S., MacPherson, S.E., Phillips, L.H., Spinnler, H., 2003. How many camels are there in Italy? Cognitive estimates standardised on the Italian population. Neurol. Sci. 24, 10-15.

Douaud, G., Filippini, N., Knight, S., Talbot, K., Turner, M.R., 2011. Integration of structural and functional magnetic resonance imaging in amyotrophic lateral sclerosis. Brain 134, 3470-3479.

Ellis, C.M., Simmons, A., Jones, D.K., Bland, J., Dawson, J.M., Horsfield, M.A. Williams, S.C., Leigh, P.N., 1999. Diffusion tensor MRI assesses corticospinal tract damage in ALS. Neurology 53, 1051-1058.

Filippini, N., MacIntosh, B.J., Hough, M.G., Goodwin, G.M., Frisoni, G.B., Smith, S.M. Matthews, P.M., Beckmann, C.F., Mackay, C.E., 2009. Distinct patterns of brain activity in young carriers of the APOE-epsilon4 allele. Proc. Natl. Acad. Sci. U.S.A 106, 7209-7214.

Foerster, B.R., Callaghan, B.C., Petrou, M., Edden, R.A., Chenevert, T.L., Feldman, E.L. 2012. Decreased motor cortex gamma-aminobutyric acid in amyotrophic lateral sclerosis. Neurology 78, 1596-1600.

Folstein, M.F., Folstein, S.E., McHugh, P.R., 1975. "Mini-mental state". A practical method for grading the cognitive state of patients for the clinician. J. Psychiatr. Res. 12, 189-198.

Galantucci, S., Tartaglia, M.C., Wilson, S.M., Henry, M.L., Filippi, M., Agosta, F. Dronkers, N.F., Henry, R.G., Ogar, J.M., Miller, B.L., Gorno-Tempini, M.L., 2011 White matter damage in primary progressive aphasias: a diffusion tensor tractography study. Brain 134, 3011-3029.

Gordon, P.H., Cheng, B., Katz, I.B., Pinto, M., Hays, A.P., Mitsumoto, H., Rowland, L.P., 2006. The natural history of primary lateral sclerosis. Neurology 66, 647-653.

Grace, J., Amick, M.M., D’Abreu, A., Festa, E.K., Heindel, W.C., Ott, B.R., 2005. Neuropsychological deficits associated with driving performance in Parkinson's and Alzheimer's disease. J. Int. Neuropsychol. Soc. 11, 766-775.

Iwata, N.K., Kwan, J.Y., Danielian, L.E., Butman, J.A., Tovar-Moll, F., Bayat, E. Floeter, M.K., 2011. White matter alterations differ in primary lateral sclerosis and amyotrophic lateral sclerosis. Brain 134, 2642-2655.

Jelsone-Swain, L.M., Fling, B.W., Seidler, R.D., Hovatter, R., Gruis, K., Welsh, R.C., 2010. Reduced interhemispheric functional connectivity in the motor cortex during rest in limb-onset amyotrophic lateral sclerosis. Front. Syst. Neurosci. 4, 1-8.

Konagaya, M., Sakai, M., Matsuoka, Y., Konagaya, Y., Hashizume, Y., 1998. Upper motor neuron predominant degeneration with frontal and temporal lobe atrophy. Acta. Neuropathol. 96, 532-536.

Kosaka, T., Fu, Y.J., Shiga, A., Ishidaira, H., Tan, C.F., Tani, T., Koike, R., Onodera, O., Nishizawa, M., Kakita, A., Takahashi, H., 2012. Primary lateral sclerosis: uppermotor-predominant amyotrophic lateral sclerosis with frontotemporal lobar degeneration-immunohistochemical and biochemical analyses of TDP-43. Neuropathology 32, 373-384.

Kuipers-Upmeijer, J., de Jager, A.E., Hew, J.M., Snoek, J.W., van Weerden, T.W., 2001 Primary lateral sclerosis: clinical, neurophysiological, and magnetic resonance findings. J. Neurol. Neurosurg. Psychiatry 71, 615-620.

Laiacona, M., Inzaghi, M.G., De Tanti, A., Capitani, E., 2000. Wisconsin card sorting test: a new global score, with Italian norms, and its relationship with the Weigl sorting test. Neurol. Sci. 21, 279-291.

Le Forestier, N., Maisonobe, T., Piquard, A., Rivaud, S., Crevier-Buchman, L. Salachas, F., Pradat, P.F., Lacomblez, L., Meininger, V., 2001. Does primary lateral sclerosis exist? A study of 20 patients and a review of the literature. Brain 124, 1989-1999.

Li, G., Pleasure, S.J., 2011. Exciting information for inhibitory neurons. Neuron 69, $585-587$.

Lloyd, C.M., Richardson, M.P., Brooks, D.J., Al-Chalabi, A., Leigh, P.N., 2000. Extramotor involvement in ALS: PET studies with the GABA(A) ligand [(11)C]flumazenil. Brain 123, 2289-2296. 
Luo, C., Chen, Q., Huang, R., Chen, X., Chen, K., Huang, X., Tang, H., Gong, Q., Shang, H.F., 2012. Patterns of spontaneous brain activity in amyotrophic lateral sclerosis: a resting-state FMRI study. PLoS One 7, e45470.

Maekawa, S., Al-Sarraj, S., Kibble, M., Landau, S., Parnavelas, J., Cotter, D., Everall, I., Leigh, P.N., 2004. Cortical selective vulnerability in motor neuron disease: a morphometric study. Brain 127, 1237-1251.

Miceli, G., Laudanna, A., Burani, C., Capasso, R., 1994. Batteria per l'Analisi del Deficit Afasico. B.A.D.A. [B.A.D.A. A Battery for the Assessment of Aphasic Disorders]. CEPSAG, Roma.

Mochizuki, A., Komatsuzaki, Y., Iwamoto, H., Shoji, S., 2004. Frontotemporal dementia with ubiquitinated neuronal inclusions presenting with primary lateral sclerosis and parkinsonism: clinicopathological report of an autopsy case. Acta Neuropathol. 107, 377-380.

Mohammadi, B., Kollewe, K., Samii, A., Krampfl, K., Dengler, R., Munte, T.F., 2009. Changes of resting state brain networks in amyotrophic lateral sclerosis. Exp. Neurol. 217, 147-153.

Muller, H.P., Unrath, A., Huppertz, H.J., Ludolph, A.C., Kassubek, J., 2012. Neuroanatomical patterns of cerebral white matter involvement in different motor neuron diseases as studied by diffusion tensor imaging analysis. Amyotroph. Lat. Scler. 13, 254-264.

Nichols, T.E., Holmes, A.P., 2002. Nonparametric permutation tests for functional neuroimaging: a primer with examples. Hum. Brain Mapp. 15, 1-25.

Novelli, G., Papagno, C., Capitani, E., Laiacona, N., Vallar, G., Cappa, S.F., 1986. Tre test clinici di ricerca e produzione lessicale. Taratura su soggetti normali. Arch. Psicol. Neurol. Psychiatry 47, 477-506.

Orsini, A., Grossi, D., Capitani, E., Laiacona, M., Papagno, C., Vallar, G., 1987. Verbal and spatial immediate memory span: normative data from 1355 adults and 1112 children. Ital. J. Neurol. Sci. 8, 539-548.

Petri, S., Krampfl, K., Hashemi, F., Grothe, C., Hori, A., Dengler, R., Bufler, J., 2003. Distribution of GABAA receptor mRNA in the motor cortex of ALS patients, J. Neuropathol. Exp. Neurol. 62, 1041-1051.

Piquard, A., Le Forestier, N., Baudoin-Madec, V., Delgadillo, D., Salachas, F., Pradat, P.F., Derouesne, C., Meininger, V., Lacomblez, L., 2006. Neuropsychological changes in patients with primary lateral sclerosis. Amyotroph. Lat. Scler. 7, 150-160.

Pringle, C.E., Hudson, A.J., Munoz, D.G., Kiernan, J.A., Brown, W.F., Ebers, G.C., 1992. Primary lateral sclerosis. Clinical features, neuropathology and diagnostic criteria. Brain 115, 495-520.

Rascovsky, K., Hodges, J.R., Knopman, D., Mendez, M.F., Kramer, J.H., Neuhaus, J., van Swieten, J.C., Seelaar, H., Dopper, E.G., Onyike, C.U., Hillis, A.E., Josephs, K.A., Boeve, B.F., Kertesz, A., Seeley, W.W., Rankin, K.P., Johnson, J.K., GornoTempini, M.L., Rosen, H., Prioleau-Latham, C.E., Lee, A., Kipps, C.M., Lillo, P., Piguet, O., Rohrer, J.D., Rossor, M.N., Warren, J.D., Fox, N.C., Galasko, D., Salmon, D.P., Black, S.E., Mesulam, M., Weintraub, S., Dickerson, B.C., DiehlSchmid, J., Pasquier, F., Deramecourt, V., Lebert, F., Pijnenburg, Y., Chow, T.W., Manes, F., Grafman, J., Cappa, S.F., Freedman, M., Grossman, M., Miller, B.L., 2011. Sensitivity of revised diagnostic criteria for the behavioural variant of frontotemporal dementia. Brain 134, 2456-2477.

Riva, N., Falini, A., Inuggi, A., Gonzalez-Rosa, J.J., Amadio, S., Cerri, F., Fazio, R., Del Carro, U., Comola, M., Comi, G., Leocani, L., 2012. Cortical activation to voluntary movement in amyotrophic lateral sclerosis is related to corticospinal damage: electrophysiological evidence. Clin. Neurophysiol. 123, 1586-1592.

Smith, S.M., Fox, P.T., Miller, K.L., Glahn, D.C., Fox, P.M., Mackay, C.E., Filippini, N., Watkins, K.E., Toro, R., Laird, A.R., Beckmann, C.F., 2009. Correspondence of the brain's functional architecture during activation and rest. Proc. Natl. Acad. Sci. U.S.A 106, 13040-13045.
Smith, S.M., Nichols, T.E., 2009. Threshold-free cluster enhancement: addressing problems of smoothing, threshold dependence and localisation in cluster inference. Neuroimage 44, 83-98.

Spinnler, H., Tognoni, G., 1987. Standardizzazione e taratura Italiana di test neuropsicologici. Ital. J. Neurol. Sci. 6, 1-120.

Strong, M.J., Grace, G.M., Freedman, M., Lomen-Hoerth, C., Woolley, S. Goldstein, L.H., Murphy, J., Shoesmith, C., Rosenfeld, J., Leigh, P.N., Bruijn, L Ince, P., Figlewicz, D., 2009. Consensus criteria for the diagnosis of frontotemporal cognitive and behavioural syndromes in amyotrophic lateral sclerosis. Amyotroph. Lat. Scler. 10, 131-146.

Tan, C.F., Kakita, A., Piao, Y.S., Kikugawa, K., Endo, K., Tanaka, M., Okamoto, K. Takahashi, H., 2003. Primary lateral sclerosis: a rare upper-motorpredominant form of amyotrophic lateral sclerosis often accompanied by frontotemporal lobar degeneration with ubiquitinated neuronal inclusions? Report of an autopsy case and a review of the literature. Acta Neuropathol. 105, 615-620.

Tartaglia, M.C., Laluz, V., Rowe, A., Findlater, K., Lee, D.H., Kennedy, K., Kramer, J.H. Strong, M.J., 2009. Brain atrophy in primary lateral sclerosis. Neurology 72 1236-1241.

Tedeschi, G., Trojsi, F., Tessitore, A., Corbo, D., Sagnelli, A., Paccone, A. D’Ambrosio, A., Piccirillo, G., Cirillo, M., Cirillo, S., Monsurro, M.R., Esposito, F., 2010. Interaction between aging and neurodegeneration in amyotrophic lateral sclerosis. Neurobiol. Aging 33, 886-898.

Turner, M.R., Cagnin, A., Turkheimer, F.E., Miller, C.C., Shaw, C.E., Brooks, D.J. Leigh, P.N., Banati, R.B., 2004. Evidence of widespread cerebral microglial activation in amyotrophic lateral sclerosis: an [11C](R)-PK11195 positron emission tomography study. Neurobiol. Dis. 15, 601-609.

Turner, M.R., Hammers, A., Al-Chalabi, A., Shaw, C.E., Andersen, P.M., Brooks, D.J. Leigh, P.N., 2007. Cortical involvement in four cases of primary lateral sclerosis using [(11)C]-flumazenil PET. J. Neurol. 254, 1033-1036.

Turner, M.R., Kiernan, M.C., 2012. Does interneuronal dysfunction contribute to neurodegeneration in amyotrophic lateral sclerosis? Amyotroph. Lat. Scler. 13 245-250.

Unrath, A., Muller, H.P., Riecker, A., Ludolph, A.C., Sperfeld, A.D., Kassubek, J., 2010. Whole brain-based analysis of regional white matter tract alterations in rare motor neuron diseases by diffusion tensor imaging. Hum. Brain Mapp. 31 1727-1740.

van der Graaff, M.M., Sage, C.A., Caan, M.W., Akkerman, E.M., Lavini, C., Majoie, C.B., Nederveen, A.J., Zwinderman, A.H., Vos, F., Brugman, F., van den Berg, L.H., de Rijk, M.C., van Doorn, P.A., Van Hecke, W., Peeters, R.R., Robberecht, W. Sunaert, S., de Visser, M., 2011. Upper and extra-motoneuron involvement in early motoneuron disease: a diffusion tensor imaging study. Brain 134 1211-1228.

Verstraete, E., van den Heuvel, M.P., Veldink, J.H., Blanken, N., Mandl, R.C., Hulshoff Pol, H.E., van den Berg, L.H., 2010. Motor network degeneration in amyotrophic lateral sclerosis: a structural and functional connectivity study. PLoS One 5 e13664.

Vucic, S., Nicholson, G.A., Kiernan, M.C., 2008. Cortical hyperexcitability may precede the onset of familial amyotrophic lateral sclerosis. Brain 131, 1540-1550.

Wittstock, M., Wolters, A., Benecke, R., 2007. Transcallosal inhibition in amyotrophic lateral sclerosis. Clin. Neurophysiol. 118, 301-307.

Zago, S., Poletti, B., Corbo, M., Adobbati, L., Silani, V., 2008. Dysgraphia in patients with primary lateral sclerosis: a speech-based rehearsal deficit? Behav. Neurol 19, 169-175. 